\title{
Effects of Repeated Training on Controlled Force Exertion and Retention in Dominant and Nondominant Hands
}

\author{
H. Kubota ${ }^{1, *}$, S. Demura², M. Uchiyama ${ }^{3}$, K. Takahashi ${ }^{4}$ \\ ${ }^{1}$ Faculty of Education, Gifu University, Gifu, Japan \\ ${ }^{2}$ Graduate School of Natural Science \& Technology, Kanazawa University, Ishikawa, Japan \\ ${ }^{3}$ Research and Education Center for Comprehensive Science, Akita Prefectural University, Akita, Japan \\ ${ }^{4}$ Faculty of Community Health Care, Department of Judo Physical Therapy, Teikyo Heisei University, Chiba, Japan \\ *Corresponding author: hkubota@gifu-u.ac.jp
}

Received February 19, 2014; Revised February 27, 2014; Accepted March 19, 2014

\begin{abstract}
This study aimed to examine the effects of repeated training on the controlled force exertion (CFE) test and retention in dominant and nondominant hands. The subjects were 10 young males (mean age, $21.8 \pm 1.4$ years). The CFE test was used as the repeated training task and performed 5 days per week over 3 weeks (a total of 15 times). The exertion value of the subjects' handgrip strengths was matched to constantly changing demand values for $40 \mathrm{~s}$. Three trials were performed after one practice trial. The difference between the demand value and the grip exertion value was used as the evaluation parameter, referred to as the CFE error, and the mean of the second and third trials was used for analysis. The initial and final values for the dominant and nondominant hands and those taken one month after the repeated training task were used for analysis. A two-way analysis of variance revealed that the final CFE errors for both hands were significantly smaller than the initial CFE errors, and no significant difference was found between the dominant and nondominant hands in the final tests. In addition, after one month, the CFE errors for both hands were significantly smaller than the initial CFE errors and were significantly smaller in the dominant hand than in the nondominant hand. Repeated training in controlled force exertion improves CFE in both the dominant and nondominant hands, but the dominant and nondominant hands differ in retention of the effects.
\end{abstract}

\section{Keywords: laterality, motor control, motor learning}

Cite This Article: H. Kubota, S. Demura, M. Uchiyama, and K. Takahashi, "Effects of Repeated Training on Controlled Force Exertion and Retention in Dominant and Nondominant Hands." American Journal of Sports Science and Medicine, vol. 2, no. 4 (2014): 132-135. doi: 10.12691/ajssm-2-4-3.

\section{Introduction}

The functional right-left difference in body parts with bilateral symmetry in humans is called "laterality" $[1,2,3,4]$. In laterality, either the right or left side in a body with bilateral symmetry is superior to the other side in achievement of motor or cognitive task. The dominant hand is generally superior in muscle strength, quickness, accuracy, and dexterity. Laterality appears from infancy because of the influence of inherited factors [5]. The difference between the dominant and nondominant hands becomes more pronounced because of the preferential use of the dominant hand in activities of daily life such as writing or using chopsticks. Laterality also influences the coordination ability. Noguchi et al. [6] reported that laterality is found in the Moving Beans with Tweezers test and the Purdue Pegboard test, which are used to evaluate dexterity, an element of coordination; their results indicated that the dominant hand is superior to the nondominant hand. Ohtsuki et al. [7] found that laterality in grading ability becomes more pronounced with the influence of an acquired factor.

Controlled force exertion (CFE) refers to the ability to properly adjust force exertion values to demand values. To evaluate CFE, Nagasawa et al. [8,9] developed a test in which the subjects match their submaximal grip strengths to changing demand values presented as a bar chart or a waveform on the display of a personal computer. Kubota et al. [11,12] examined laterality of CFE in young males and females using the CFE test and reported that it is found in both males and females. In addition, they examined and reported laterality in young and elderly females.

As mentioned above, lateral dominance appears in motor tasks that demand dexterity of the hands, fingers, and upper limbs, and the dominant hand is superior [13]. Taylor and Heilman [14] examined the differences between the right and left hands in motor task proficiency using a complex key-pressing task and reported that the motor task proficiency period is shorter in the right hand than in the left hand. It can be inferred that the dominant hand, which is preferentially used in the activities of daily 
life, becomes more proficient at functions that involve a series of movements than the nondominant hand. The dominant hand is also superior in functions involving the peripheral and central nervous systems; thus, the movements improve smoothly with repetition of the appropriate feedback.

Regarding the retention of motor learning, Shea and Kohl $[15,16]$ examined constant and variable practice using the handgrip task and reported the latter superior for retention of motor learning. However, retention of motor learning in the dominant and nondominant hands and retention of the effects of repeated CFE training have not previously been examined. Because the dominant hand is superior to the nondominant hand in motor learning [14], a difference between the two hands may be found in retention of motor learning.

It was therefore hypothesized that laterality would be found in retention of motor learning in addition to the training effect of the CFE test. This study aimed to examine the effects of repeated CFE test training and the retention of the effects in the dominant and nondominant hands.

\section{Methods}

\subsection{Subjects}

Subjects were 10 healthy young males (mean age 21.8 \pm 1.4 years; height $171.8 \pm 5.8 \mathrm{~cm}$; weight $65.2 \pm 4.7 \mathrm{~kg}$ ). Mean values for height and body mass were similar to the Japanese normative values (Laboratory Physical Education in Tokyo Metropolitan University, 1989). Before the experiment, all subjects were judged to be right-handed by a Demura et al. [17] handedness inquiry. No subject had any disability of the upper limbs. Each subject could observe the computer display without difficulty; therefore, it was judged that individual vision did not affect the measurements. Prior to measurement, the purposes and procedures of this study were explained in detail to each subject, and consent for participation in this study was obtained from all subjects. The protocol for this study was approved by the Ethics Committee on Human Experimentation of the Faculty of Human Science, Kanazawa University (Ref. No. 2012-02).

\subsection{Measurement}

\subsubsection{Experimental Procedure}

The subjects performed repeated training (a total of 15 times) of controlled force exertion (4 trials per day by the dominant and nondominant hands) 5 days per week for 3 weeks. Repeated training in controlled force exertion was conducted according to the same procedure as the CFE test, and CFE errors were recorded each time. The subjects were not restricted to perform activities of daily life.

\subsubsection{Controlled force exertion test}

We measured maximal handgrip strength and CFE using a Smedley's handgrip mechanical dynamometer (Sakai, Tokyo, Japan) with a range of 0.0-99.9 kg and a hand biofeedback system (EG-100; Sakai, Tokyo, Japan). The information from the handgrip device was transmitted at a sampling rate of $20 \mathrm{~Hz}$ to a computer through a data output cable after A/D conversion. The subjects exerted grip strength using a handgrip device while sitting on a chair in front of the computer display, with the elbow straight and close to the body, without contact between the dynamometer and the body or the chair. The size of the grip was set such that they felt comfortable squeezing it. They performed the CFE test while attempting to minimize the differences between the demand and grip values that were being presented on the computer. A bar chart was used to represent the data according to the criteria established by Nagasawa et al. [9]. The demand values increased and decreased from $5 \%$ to $25 \%$ of the maximal grip strength at a constant frequency of $0.2 \mathrm{~Hz}$. Relative values based on the maximal handgrip strength, but not absolute values, were used as the demand values. First, the maximal handgrip strength was measured twice with a 1-min interval to set the demand values, and the greater value was used in this study. The program was designed to present the demand values within a constant range on the display regardless of differences in each participant's maximal handgrip strength. The duration of the CFE test was $40 \mathrm{~s}$, and the CFE was estimated using the data, excluding the first $15 \mathrm{~s}$ of each trial, considering a stable time of performance. The sum of the differences between the demand value and the grip exertion value was used as the estimates of the CFE error. Smaller errors in CFE were interpreted to indicate superior CFE. The subjects performed the CFE test 3 times after one practice trial, and the mean of the second and third trials was used as a representative value.

\subsection{Statistical Analysis}

The data were reported using ordinary statistical methods, including mean and standard deviation $[ \pm$ standard deviation (SD)]. A two-way analysis of variance (dominant/nondominant hands and measurement period) was used to examine significant differences between the means of the CFE errors in the dominant and nondominant hands. When a significant interaction or a primary effect was found, a multiple comparison test was performed using Tukey's Honestly Significant Difference method. The level of significance was set a priori to 0.05. An effect size (ES) was calculated to examine the size of the mean difference. ES was interpreted as follows: $<0.2$ as small, $>0.5$ as intermediate, and $>0.8$ as large.

\section{Results}

Table 1 shows the means and SD of the CFE errors for the dominant and nondominant hands at the initial and final times and one month after repeated training. Twoway analysis of variance revealed a significant primary effect in the dominant/nondominant hands and the measurement period. Multiple comparisons showed that the dominant hand had smaller CFE errors than the nondominant hand at the initiation of the experiment (ES $=0.46$ ). No difference was found between both hands at the time of the final measurement. One month after the repeated training, the CFE errors in the dominant hand were smaller than that in the nondominant hand (ES = 0.56). In both hands, the errors at the initial measurement were larger than those at the final measurement (dominant 
hand: $\mathrm{ES}=2.34$, nondominant hand: $\mathrm{ES}=2.92$ ). Similarly, in both hands, the errors at the initial time were larger than those one month after the end of repeated training (dominant hand: $\mathrm{ES}=2.04$, nondominant hand: $\mathrm{ES}=$ 2.35).

Table 1. Means of the CFE errors in the dominant and nondominant hands and testresults (two-way analysis of variance)

\begin{tabular}{|c|c|c|c|c|c|c|c|c|c|c|}
\hline & \multicolumn{3}{|c|}{ Dominant hand } & \multicolumn{3}{|c|}{ Nondominant hand } & & \multirow[b]{2}{*}{ F-value } & \multirow[b]{2}{*}{ Partal $\eta^{2}$} & \multirow{2}{*}{$\begin{array}{c}\text { Post hoc } \\
\text { HSD }\end{array}$} \\
\hline & Mean & SD & $\mathrm{CV}$ & Mean & SD & $\mathrm{CV}$ & & & & \\
\hline Initial time & 569.1 & 150.1 & 26.4 & 632.3 & 126.7 & 20.0 & F1 & $22.03^{*}$ & 0.71 & Initial, One month: Dom < Nondom \\
\hline Final time & 296.6 & 67.7 & 22.8 & 327.0 & 76.8 & 23.5 & $\mathrm{~F} 2$ & $74.98 *$ & 0.89 & Dom, Nondom: Initial > Final, One month \\
\hline One month after training & 326.7 & 75.2 & 23.0 & 372.9 & 90.8 & 24.3 & F3 & 0.37 & 0.04 & \\
\hline
\end{tabular}

unit: \%, ${ }^{*}$ p < 0.05, F1: dominant and non-dominant hand, F2: time, F3: intaraction, SD: standard deviation, CV: coefficient of variance, CV = SD/mean $\times$ 100, Dom: Dominant hand, Nondom: Nondominant hand, Initial: Initial time, Final: Final time, One month: One month after training

\section{Discussion}

The results of the present study showed that in both the dominant and nondominant hands, the CFE errors improved by repeated training. Nakamura et al. [18] reported that the learning effect in tracking tasks is related to both comprehension of target trajectory (declarative memory) and improvement of the target tracking procedure (procedural memory). It can be inferred that improvement of these two types of memory promotes learning, thereby decreasing the CFE errors, because the CFE test performed in this study used the same relative load and the same speed in each trial during training. In the CFE test, visual and perceptual information from peripheral tissues is processed in the brain, and muscle strength is exerted in response to motor commands from the brain. In short, the subjects consider the size of the error between the demanded and exerted values based on visual feedback and coordinate force output via motor commands [12]. Thus, the accuracy of cognitive information processing, motor commands, and force output improved with repeated training.

The present results indicate that the difference between the dominant and nondominant hands detected at the initiation of the experiment was lost by 3 weeks period of the repeated training. Lateral dominance generally appears in motor tasks that require dexterity in the hands, fingers, and upper limbs and the dominant hand is superior [13]. Kubota and Demura [11,12] reported that laterality was found in young males and females and also in young and elderly females. In the present study, the CFE errors at the initiation of the experiment were also smaller in the dominant hand than in the nondominant hand.

However, the difference between the dominant and nondominant hands was lost after 3 weeks from the initiation of the experiment. Noguchi et al. [19] reported that lateral dominance is found in the practice effect in the Moving Beans with Tweezers test from results showing that the practice effect is found only in the nondominant hand. They reasoned that the practice effects was not found in the dominant hand because it is frequently used in movements similar to those in the Moving Beans with Tweezers test in daily life, and therefore did not develop. For motor tasks similar to movements in daily life, the practice effect may not be evident in the dominant hand after related coordination skills improve to a certain level. From the above, it can be inferred that the difference between the dominant and nondominant hands reduced because the performance of the nondominant hand improved, while the performance of the dominant hand reached an upper limit.

The CFE error reduced in the dominant and nondominant hands by repeated training over a 3-week period and the effects remained one month after training. However, the difference between the CFE of the two hands at the initiation of the experiment reappeared one month after the training, perhaps because the practice effect may fade more easily in the nondominant hand than the dominant hand.

\section{Conclusion}

In both the dominant and nondominant hands, CFE is improved by repeated training in controlled force exertion. The dominant and nondominant hands may retain the effects of training differently because the training effect erases the difference in CFE between the two hands and it reappears one month after the task.

\section{References}

[1] Geshwind, N., Behan, P., "Left-handedness: Association with immune disease, migraine, and developmental learning disorder," Proc Natl Acad Sci, 79. 5097-5100. 1982.

[2] Gur, R. C., Turetsky, B. I., Matsui, M., Yan, M., Bilker, W., Hughett, P., Gur, R.E., "Sex differences in brain gray and white matter in health young adults: Correlations with cognitive performance," J Neurosci, 19. 4065-4072. 1999.

[3] Dolcos, F., Rice, H.J., Cabeza, R., "Hemispheric asymmentry and aging: right hemisphere decline or asymmetry reduction," Neurosci Biobehav Rev, 26. 819-825. 2002.

[4] Roy, E.A., Bryden, P., Cavill, S., "Hand differences in pegboard performance through development,” Brain Cogn, 53. 315-317. 2003.

[5] Chi, J.G., Dooling, E.C., Gilles, F.H., "Left-right asymmetry of the temporal speech areas of the human fetus," Arch Neurol, 34. 346-348. 1977.

[6] Noguchi, T., Demura, S., Aoki, H., "Superiority of dominant and nondominant hands in static strength and controlled force exertion,” Percept Mot Skills, 109. 339-346. 2009.

[7] Ohtsuki, H., Hasebe, S., Okano, M., Furuse, T., “Comparison of surgical results of responders and non-responders to prism adaptation test in intermittent exotropia," Acta Ophthalmol Scand, 75. 528-531. 1997.

[8] Nagasawa, Y., Demura, S., Yamaji, S., Kobayashi, H., Matsuzawa, J., "Ability to coordinate exertion of force by the dominant hand: comparisons among university students and 65-to 78-year-old men and women,” Percept Mot Skills, 90. 995-1007. 2000.

[9] Nagasawa, Y., Demura, S., "Development of an apparatus to estimate coordinated exertion of force," Percept Mot Skills, 94. 899-913. 2002.

[10] Nagasawa, Y., Demura, S., Kitabayashi, T., "Concurrent validity of tests to measure the coordinated exertion of force by 
computerized target pursuit,” Percept Mot Skills, 98(2), 551-560. 2004.

[11] Kubota, H., Demura, S., "Gender differences and laterality in maximal handgrip strength and controlled force exertion in young adults," Health, 3(11). 684-688. 2011.

[12] Kubota, H., Demura, S., Kawabata, H., "Laterality and Age-level Differences between young women and elderly women in controlled force exertion (CFE)," Arch Gerontol Geriatr, 54. e68e72. 2012.

[13] Demura, S., Yamaji, S., Goshi, F., Nagasawa, Y., "Lateral dominance of legs in maximal muscle power, muscular endurance, and grading ability," Percept Mot Skills, 93. 11-23. 2001.

[14] Taylor, H. G., Heilman K. M., "Left-hemisphere motor dominance in right-handers," Cortex, 16. 587-603. 1980.

[15] Shea, C.H., Kohl, R.M., "Specificity and variability of practice," Res Q Exerc Sport, 61. 169-177. 1990.
[16] Shea, C. H., Kohl, R. M., "Composition of practice: Influence on the retention of motor skills,” Res Q Exerc Sport, 62. 187-195. 1991.

[17] Demura, S., Sato, S., Nagasawa, Y., "Re-examination of useful items for determining hand dominance," Gazz Med Ital-Arch Sci Med, 168(3). 169-177. 2009.

[18] Nakamura, M., Ide, J., Sugi, T., Terada, K., Shibasaki, H., "Method for studying learning effect on manual tracking of randomly moving visual trajectory and its application to normal subjects," IEICE, J78-D-II, (3). 547-558. 1995.

[19] Noguchi, T., Demura, S., Nagasawa, Y., Uchiyama, M., “An examination of practice and laterality effect on the purdue pegboard and moving beans with tweezers," Percept Mot Skills, 102. 265-274. 2006 . 